\title{
Improved Spatial Resolution of EDX/SEM for the Elemental Analysis of Nanoparticles.
}

\author{
Johannes Mielke ${ }^{1}$, Steffi Rades $^{1},{\text { Erik } \text { Ortel }^{1}, \text { Tobias Salge }^{2} \text { and Vasile-Dan Hodoroaba }}^{1}$ \\ 1. BAM Federal Institute for Materials Research and Testing, Division 6.8 Surface Analysis and \\ Interfacial Chemistry, 12200 Berlin, Germany. \\ 2. Natural History Museum, Core Research Laboratories, Cromwell Road, SW7 5BD London, United \\ Kingdom.
}

The interest in nanoparticles remains at a high level in fundamental research since many years and increasingly, nanoparticles are incorporated into consumer products to enhance their performance. Consequently, the accurate and rapid characterization of nanoparticles is more and more demanded. Electron microscopy (SEM, TSEM and TEM) is one of the few techniques which are able to image individual nanoparticles. It was demonstrated recently that the transmission electron microscopy at a SEM can successfully be applied as a standard method to characterize accurately the size (distribution) and shape of nanoparticles down to less than $10 \mathrm{~nm}[1,2]$.

In many cases a (nano)material consists of particles having a similar size (and form) with varying elemental composition. In this contribution, some case studies are presented, demonstrating the chemical sensitivity of modern EDX systems at the nano-scale, enabling a distinction between nanoparticles of different chemical composition. In order to reach the necessary high spatial resolution of the EDX signals, two prerequisites should be fulfilled: the operation of the transmission mode at SEM, i.e. preparation of the (nano)material on TEM grids, as well as the use of highly-sensitive EDS detectors with increased solid angle to compensate for the low signal-to-noise EDX spectra emitted by nanoparticles.

Besides pure nanoparticles with a size well below $100 \mathrm{~nm}$, and nanoparticle mixtures, also nanoparticles with a more complicated form and chemical composition such as core-shell nanoparticles are investigated by high-resolution TSEM/EDX [3]. Also the detection of contaminants/impurities becomes possible up to a certain scale.

In order to check the attended EDX spatial resolution by using various high-sensitive EDS systems in conjunction with T-SEM, dedicated experiments have been carried out. The lack of reference nanomaterials with certified size and chemical composition is well-known. One of the few examples is the reference sample BAM-L200, a material with stripe structures of certified dimensions in the nano-range, specially designed for testing the spatial resolution of imaging micro/nano-probe techniques. This material has been prepared as a FIB lamella [4] and it was demonstrated that spatial resolutions of the EDX signals in the range of a few nm can be achieved.

The need for and the availability of automatic image acquisition (including spectral information) with the goal of automatic chemical classification will be discussed. The coupled automatic data evaluation will be also addressed by some practical examples. 


\section{References:}

[1] E Buhr, N Senftleben, T Klein et al., Meas. Sci. Technol. 20 (2009), 084025 (9 pp.).

[2] V-D Hodoroaba, C Motzkus, T Macé et al., Microsc. Microanal. 20 (2014), p. 602.

[3] S Rades, V-D Hodoroaba, T Salge et al., RSC Adv. 4 (2014), p. 49577.

[4] M Senoner, A Maaßdorf, H Rooch et al., Anal Bioanal Chem, DOI 10.1007/s00216-014-8135-7, in press.

[5] The research leading to these results has received funding from the European Union's Seventh Framework Programme (FP7/2007-2013) under grant agreements $n^{\circ} 604347$ (NanoDefine) and $n^{\circ}$ 263147 (NanoValid).
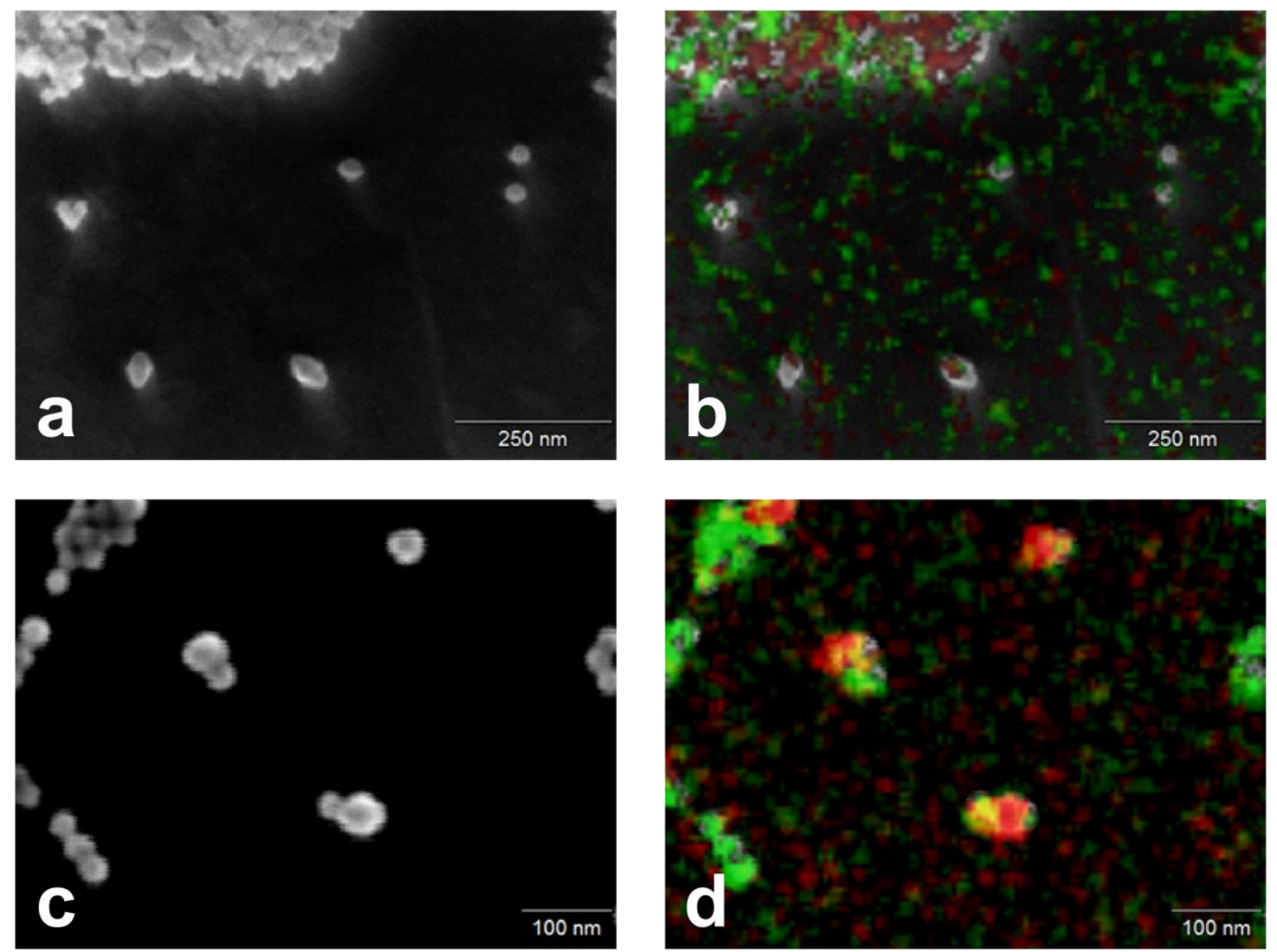

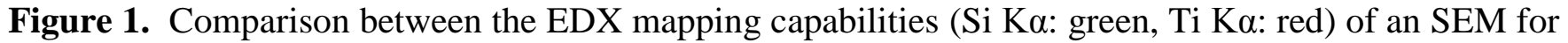
a mixture of $\mathrm{SiO}_{2}$ and $\mathrm{TiO}_{2}$ nanoparticles on bulk graphite $(\mathrm{a}, \mathrm{b})$ and on a thin, electron transparent TEM grid (c, d). On the bulk substrate, the different chemical composition of the individual nanoparticles cannot be resolved due to the intense substrate background, whereas on the TEM grid, the EDX mapping (with the same EDS detector) shows a clear distinction between the different nanoparticle types, which is possible due to the largely reduced background intensity. 